\title{
Koselleck, Reinhart, und Carl Schmitt (2019): Der Briefwechsel 1953-1983. Herausgegeben von Jan Eike Dunkhase
}

\author{
Berlin: Suhrkamp. 459 Seiten. $42 €$
}

\section{Sebastian Huhnholz}

Online publiziert: 17. April 2020

(C) Der/die Autor(en) 2020

Der von Jan Eike Dunkhase editierte und 1953 einsetzende Briefwechsel zwischen dem durch seine nationalsozialistische Kompromittierung berüchtigten Staatsrechtslehrer Carl Schmitt und dem anfangs noch über „Kritik und Krise“ promovierenden Historiker Reinhart Koselleck wirft viel Licht auf eine seit nun 60 Jahren von Legenden umnebelte Beziehung. Das Gespür des nach dem Nationalsozialismus mit Lehrverbot belegten Schmitt für junge Talente und die ausgesucht kluge Höflichkeit des von Krieg und Gefangenschaft geprägten Koselleck griffen günstig ineinander. Die nun endlich veröffentlichte Korrespondenz macht deutlich, dass der später berühmte Begriffshistoriker Koselleck schon von Beginn der Bekannt- und baldigen Freundschaft im Jahr 1950 an ein eigenes Programm vertritt und dabei von Schmitt - anders als es Teile der bisherigen Rezeption unterstellen - weniger ge- oder gar verführt als begleitet und beraten wird.

Bereits der erste, von der bisherigen Forschung bereits viel beachtete Brief des jungen Koselleck lässt den Herausgeber Dunkhase treffend resümieren, dass „Denkprozesse von Jahren hier in fast schwindelerregender Verdichtung“ aufstoßen (S. 418). Kosellecks fachkritische Sentenz etwa, dass ,,der Abbau der fortschrittlichen Zukunft [...] die Historie nicht davor bewahrt, eine linienhafte Vergangenheit beizubehalten“ (S. 11), liest sich gleichsam wie ein Fazit der erst kommenden Dissertation „Kritik und Krise“ wie auch als ein Aphorismus zu vielen erst späteren Geschichtstheoriefragmenten. Der jeder Geschichtsphilosophie gegenüber äußerst skeptische Koselleck wehrte sich also schon früh gegen betont gegenwartsdienliche Interpretationen der Historie, gegen allzu ideologische Indienstnahme von Vergangenheit. Dagegen bestand er auf der systematischen Reflexion ,,vergangener Zukunft“, auf die prinzipielle Kontingenz politischen Handelns.

S. Huhnholz $(\bowtie)$

Institut für Politikwissenschaft, Gottfried Wilhelm Leibniz Universität Hannover, Hannover,

Deutschland

E-Mail: s.huhnholz@ipw.uni-hannover.de 
Wie für Schmittiana (und mittlerweile auch „Koselleckiana“) typisch, lassen sich werk-, personen- und zeitgeschichtliche Vielfalt dieses über vier Jahrzehnte bis fast zu Schmitts Tod reichenden Austauschs hier kaum würdigen. Hinzu kommt, dass der Briefwechsel nicht alles ist. Besuche, Netzwerke, Tagebücher und Telefonate sind mitzudenken. Dass die Edition des Schriftverkehrs eine Konzentration auf Nachlassteile verlangt, ermöglicht dennoch diverse Klärungen. Und Bewunderung für den Herausgeber (der u.a. mit einer Biografie über Kosellecks Vorgesetzten, Kollegen und Mitherausgeber der „Geschichtlichen Grundbegriffe" Werner Conze hervortrat) werden vor allem jene nicht verwehren, die um die enigmatischen Handschriftbilder Schmitts und Kosellecks wissen oder die Quellenvielfalt einmal in Augenschein nehmen konnten. Neben den formalen Briefen zählen Widmungen, Glossen, Postkarten, Zeichnungen, mehrfarbige Notizen aus verschiedenen Zeiten u. v.a.m. zum Inventar der Nachlässe.

Der Band setzt direkt mit dem Briefwechsel ein. Inhaltliche Bezüge, Namen usw. werden nach jeder Einzelpost sparsam erklärt, sodass auch für Unvertraute schnell ein Bild entsteht. Editorische Notizen, Bestandsverzeichnisse und Register vollenden das Buch. Schwarzweiße Abbildungen ausgesuchter Dokumente (Widmungen, Postkarten etc.) ermöglichen Eindrücke der äußerlichen Formenvielfalt. Weitere Materialien (darunter Schmitts Briefe an Kosellecks Gattin und einige Interviews Kosellecks zu Schmitt) finden sich ebenfalls wiederveröffentlicht.

Das unter der Leitformel „Asymmetrische Korrespondenz“ stehende Nachwort des Herausgebers hält Biografisches knapp und informiert elegant über die Beziehung. Auffälligkeiten wie Unbestimmbares werden benannt, beispielsweise zeitliche Schwerpunkte des nicht durchgehend engen Kontakts erklärt. Dabei enthält sich Dunkhase eines ausgreifenden Kontextualismus, verzichtet auf eigene oder fremde Deutungen und fundiert das schmale Nachwort auf einer geschickten Auswahl von Forschungsliteratur.

Da über Schmitt eher zu viele als zu wenige Informationen und Meinungen im Umlauf sind, ist solch zurückhaltender Überlieferungsbefund wohltuend. In Kosellecks Fall indes entkommt der Herausgeber nicht dem Problem, dass die Forschung viele Faktizitätsvermutungen aus dem vertrauten Umfeld und insbesondere den Eigendarstellungen bezieht (oder beziehen muss), mit denen der im Alter auch als Zeitzeuge gefragte Koselleck rückblickend Auskunft gab.

Viele von Kosellecks Berichten werden durch die Korrespondenzquellen freilich gedeckt. Und sie erübrigen die Koselleck eigene Bescheidenheit. Dunkhase unterstreicht, dass „Ergebenheitsbekundungen zum Trotz“ sich schon ,der junge Koselleck stets als eigenständiger Denker“ beweist (S. 422), der „mäeutische Part, den Schmitt Koselleck gegenüber einnimmt“ (S. 419), durch ein „,unaufdringliches Selbstbewusstsein, das er Schmitt gegenüber an den Tag legt“ (S. 422), balanciert wird. Konflikte und Divergenzen mit dem baldigen Greis moderiert Koselleck ,ehrerbietig wie immer“: Schmitts ,laborierende [...] Selbstbezogenheit [...] ficht den Jüngeren nicht an“" (S. 423).

Kosellecks eigenes Werk und Wirken ist im Briefwechsel wohl auch darum weniger präsent. Das gilt sogar für das begriffsgeschichtliche Großprojekt. Über Teile des ersten, des A-D-Bandes zeigt sich Schmitt - Stichworte „Ausnahmezustand“, „Bund“, „Cäsarismus“, „Diktatur“ - empört (S. 79). Und derlei Abstimmungs- 
lücken sind umso erstaunlicher wie Koselleck sicher kein politischer Schmittianer war, aber wie kein Zweiter für einen methodologischen Schmittianismus steht, der Schmitts politisch-konkrete Denkweise programmatisch verwissenschaftlichte und semantologisch paradigmatisch machte. So mag die „Asymmetrie“ der Korrespondenz (Dunkhase) über Schmitts Nützlichkeit resymmetriert worden sein: Schmitt gefiel sich sehr in der Rolle des Mentors und Koselleck gab diesem Verlangen wohldosierte Nahrung. Die Herzlichkeit des Tons jedenfalls konnte selbst den eitlen Schmitt - der so viele Geistesgrößen zeitlebens für seine Belange instrumentalisiert hatte - nicht immer darüber hinwegtäuschen, wie souverän Koselleck sich bewegte.

Trotz aller derzeit auch massenmedial forcierten Anerkennung dafür, dass nun dieser faszinierende Briefwechsel nach vergeblichen früheren Anläufen endlich so vorzüglich vor uns liegt, wird auch dieses Projekt mit Bedenken konfrontiert werden: Geht mit der immer detaillierten Historisierung Schmitts eine womöglich zu unkritische Aufwertung einher? Wird hier die alte Bundesrepublik romantisiert? Und so fort. Sicher vermag ein solcher Briefwechsel zu Verkürzungen einladen. Doch ließe sich einerseits einwenden, dass gerade die nun präziser mögliche Kontextualisierung Reflexivität erzwingt. Eine teils bizarre Gerüchtevielfalt wird durch Dunkhases Leistung endlich auch für jene prüfbar, die akribische Arbeit in den Marbacher Katakomben oder dem Duisburger Nachlassarchiv scheuen. Doch einmal mehr fällt auch auf, was eigentlich noch fehlt und nicht nur von Koselleck vergeblich gefordert worden war: eine historisch-kritische Gesamtausgabe der Werke Schmitts.

Funding Open Access funding provided by Projekt DEAL.

Open Access Dieser Artikel wird unter der Creative Commons Namensnennung 4.0 International Lizenz veröffentlicht, welche die Nutzung, Vervielfältigung, Bearbeitung, Verbreitung und Wiedergabe in jeglichem Medium und Format erlaubt, sofern Sie den/die ursprünglichen Autor(en) und die Quelle ordnungsgemäß nennen, einen Link zur Creative Commons Lizenz beifügen und angeben, ob Änderungen vorgenommen wurden.

Die in diesem Artikel enthaltenen Bilder und sonstiges Drittmaterial unterliegen ebenfalls der genannten Creative Commons Lizenz, sofern sich aus der Abbildungslegende nichts anderes ergibt. Sofern das betreffende Material nicht unter der genannten Creative Commons Lizenz steht und die betreffende Handlung nicht nach gesetzlichen Vorschriften erlaubt ist, ist für die oben aufgeführten Weiterverwendungen des Materials die Einwilligung des jeweiligen Rechteinhabers einzuholen.

Weitere Details zur Lizenz entnehmen Sie bitte der Lizenzinformation auf http://creativecommons.org/ licenses/by/4.0/deed.de. 